\title{
Effects of Angiotensin II Type 1 Receptor Antagonist, YM358, on Cardiac Hypertrophy and Dysfunction after Myocardial Infarction in Rats
}

\author{
Tomoko TokioKa-Akagi, ${ }^{*, a}$ Akira FuJimori, ${ }^{b}$ Masayuki Shibasaki, ${ }^{b}$ Yasuko Matsuda-Satoh, ${ }^{b}$ \\ Osamu INAGAKI, ${ }^{b}$ and Isao YANAGISAWA ${ }^{b}$ \\ ${ }^{a}$ Biometrics Department, Yamanouchi Pharmaceutical Co., Ltd.; 3-17-1 Hasune, Itabashi-ku, Tokyo 174-8612, Japan: \\ and ${ }^{b}$ Institute for Drug Discovery Research, Yamanouchi Pharmaceutical Co., Ltd.; 21 Miyukigaoka, Tsukuba, Ibaraki \\ 305-8585, Japan. Received December 18, 2001; accepted April 12, 2002
}

This study was undertaken to investigate the effects of an angiotensin II type 1 receptor antagonist, YM358 (2,7-diethyl-5-[[2'(1H-tetrazol-5-yl)biphenyl-4-yl]methyl]-5H-pyrazolo $[1,5-b][1,2,4]$ triazole potassium salt monohydrate), on cardiac hypertrophy and dysfunction in rats with heart failure after myocardial infarction (MI). One day after the coronary ligation, rats were randomized, and administered YM358 or vehicle for 2, 4 or 8 weeks. In MI rats, mean blood pressure, left ventricular (LV) systolic pressure, and the first derivative of $L V$ pressure significantly decreased, and LV end-diastolic pressure (LVEDP) markedly increased after 2 to 8 week treatment of YM358. From 2 weeks after the ligation, ratios of cardiac weight and lung weight to body weight (BW) significantly increased, which indicated the progression of cardiac hypertrophy and lung congestion in MI rats. Two weeks after the ligation, YM358 did not improve LV function, although it decreased the elevated LVEDP and cardiac weights/BW ratios 8 weeks after the ligation. These results indicated that long-term treatment with YM358 improves the reduced cardiac function and reduces cardiac hypertrophy after MI, and may be useful for the treatment of congestive heart failure.

Key words angiotensin II type 1 receptor antagonist; YM358; myocardial infraction; cardiac hypertrophy; rat

Angiotensin II (Ang II) plays an important modulatory role in the cardiovascular system under basal conditions and in various pathologies such as hypertension and heart failure. ${ }^{1,2)}$ The renin-angiotensin system (RAS) and the sympathetic nervous system have been postulated to play a role in regulating cardiac cell growth. Indeed, Ang II is a potent, direct stimulant of protein synthesis and growth in cultured cardiac myocytes. $^{3,4)}$ Ang II has been implicated in the development of cardiac hypertrophy and fibrosis after myocardial infarction (MI) in humans, as well as in animal models. ${ }^{5,6}$ Inhibition of Ang II formation by angiotensin converting enzyme (ACE) inhibitors or blockade through the angiotensin II type $1\left(\mathrm{AT}_{1}\right)$ receptor by antagonists actually attenuates myocardial hypertrophy ${ }^{7,8)}$ and ventricular fibrosis. ${ }^{9)}$

Heart failure is the final form of disease following progression of cardiac hypertrophy and fibrosis caused by hypertension or valvular heart disease, or progression of ischemic heart disease such as angina or MI. ${ }^{10)}$ Rats with MI induced by left coronary artery ligation are used as experimental models representing pathophysiological alterations similar to those seen in chronic heart failure (CHF) in humans. ${ }^{1,12)}$ It is also known that ventricular hypertrophy and remodeling describe a series of pathological changes after ischemia. In MI rats, plasma Ang II concentration significantly rises 1 week after coronary ligation, and the elevation is maintained for 5 weeks. ${ }^{13)}$ Seven weeks after MI in rats, characters of LV remodeling and dysfunction are developed and the expression of cardiac $\mathrm{AT}_{1}$ receptor mRNA is enhanced. ${ }^{14)}$

YM358 (2,7-diethyl-5-[[2'(1H-tetrazol-5-yl)biphenyl-4-yl]methyl]-5H-pyrazolo [1,5-b] [1,2,4]triazole potassium salt monohydrate) is a potent, nonpeptide competitive $\mathrm{AT}_{1}$ receptor antagonist, which has been verified for its specificity on Ang II in in vitro study. ${ }^{15)}$ The efficacy of YM358 in treating hypertension is well established in animal models. ${ }^{16,17)}$ Additionally, YM358 and enalapril delayed the progression of heart failure in a rat model with cardiac volume-overload. ${ }^{18)}$
Since Ang II has been purported to be a trophic hormone which may induce the cardiac hypertrophy which is detrimental in heart failure, it would be expected that an $\mathrm{AT}_{1}$ receptor antagonist, YM358, blunts the progress of heart failure.

As obsolete MI was considered to be one of the major factors leading to CHF, YM358 was orally and chronically administered to MI rats with heart failure, and time course changes of cardiac weights and hemodynamic parameters were investigated in the present study.

\section{MATERIALS AND METHODS}

Animals Male Wistar rats (12 weeks old) were obtained from Japan SLC Inc. (Shizuoka, Japan). The rats were kept at a controlled room temperature of $22^{\circ} \mathrm{C}$ under a 12-h lightdark cycle, and provided with regular rat chow and tap water. They were used after an acclimatization period of at least $3 \mathrm{~d}$. The experiments were performed under the regulations of the Animal Ethical Committee of Yamanouchi Pharmaceutical.

Myocardial-Infarction Surgery Under anesthesia, MI surgery was performed using the techniques described previously. ${ }^{19)}$ In brief, animals were anesthetized with sodium pentobarbital $(60 \mathrm{mg} / \mathrm{kg}$ i.p.) and ventilated artificially with room air at a tidal volume of $1 \mathrm{ml} / 100 \mathrm{~g}$ body weight at a rate of $50 \mathrm{strokes} / \mathrm{min}$, using a rodent respirator (Shinano, SN480-7, Japan). A thoracotomy was performed, and the pericardium was then dissected to denude the left ventricular wall. A 4-0 silk suture was looped around the proximal left anterior descending coronary artery, and ligated tightly at a site $1-2 \mathrm{~mm}$ from its origin using the thread. After the heart was returned to its normal position, the thorax was closed. Sham-operated animals were subjected to the same surgical procedure to produce infarction as above, except that the suture around the coronary artery was not tied. Using this method, the $24 \mathrm{~h}$ mortality rates were approximately $30 \%$, 
and the infarct sizes were about $40 \%$ of the left ventricle (LV) in MI rats.

YM358 Treatment in Rats with Myocardial Infarction Nineteen sham-operated rats and 57 MI rats, which survived for $24 \mathrm{~h}$ after the surgery, were used. MI rats were randomly divided into 6 groups, and YM358 (30 mg/kg/day) or vehicle was administered once daily from the day following the operation for 2, 4 or 8 weeks. Sham-operated rats were divided into 3 groups, and vehicle was administered in the same manner as in MI rats. On the day following the last administration of YM358 or vehicle, hemodynamic parameters and cardiac weights were measured. The dose of YM358 used in this study had been effective on cardiac hypertrophy in rats with CHF induced by volume-overload. ${ }^{18)}$

Changes of Cardiac Hemodynamics and Hypertrophy in Rats with Myocardial Infarction Rats were anesthetized with sodium pentobarbital $(60 \mathrm{mg} / \mathrm{kg}$ i.p.), and a polyethylene catheter (PE-50, Clay Adams, Parsippany, NJ) was inserted into the thoracic aorta via right carotid artery to measure heart rate (HR), systolic blood pressure (SBP) and diastolic blood pressure (DBP). A disposable needle (22gauge) was inserted into LV via the diaphragm to measure left ventricular systolic pressure (LVSP) and left ventricular end-diastolic pressure (LVEDP). An amplifier receiving the signal from the ventricle was connected using a bridge to a differentiator to register the first derivative of LV pressure (LV $d P / d t_{\max }$ ). Blood pressure data were used to calculate the mean blood pressure $(\mathrm{MBP}) ; \mathrm{MBP}=\mathrm{DBP}+(\mathrm{SBP}-\mathrm{DBP}) / 3$.

After measuring hemodynamic parameters, chest cavity of the rats was opened while they were still under anesthesia. The heart was rapidly excised, and placed into ice-cold saline to remove the blood. To assess the degree of cardiac hypertrophy, LV plus septum including scar tissue, left atria (LA), right ventricle (RV) and right atria (RA) were dissected and weighed at each time point. In addition, the wet weight of lung was measured to evaluate lung congestion.

Drugs YM358 was synthesized in the Institute for Drugs Discovery Research, Yamanouchi Pharmaceutical, Japan. YM358 was suspended in $0.5 \%$ methylcellulose solution and administered by oral gavage in a volume of $5 \mathrm{ml} / \mathrm{kg}$.

Statistical Analysis All the results were expressed as means \pm S.E.M. Differences among sham-operated rats, YM358-treated and vehicle-treated MI rats were assessed by one-way analysis of variance, followed by Fisher's LSD test. Differences were considered to be statistically significant when the $p$ value was less than 0.05 .

\section{RESULTS}

Mortality Of 57 MI rats assigned for the treatment, 6 rats $(1,1$ and 2 vehicle-treated rats for 2, 4 and 8 weeks, respectively; and 2 YM358-treated rats for 4 weeks) died during the course of the study, and were excluded from the analysis. However, none of the sham-operated rats died during the study. Results were thus obtained from $51 \mathrm{MI}$ rats (5, 9 and 11 vehicle-treated rats for 2, 4 and 8 weeks, respectively; and 6, 7 and 13 YM358-treated rats for 2, 4 and 8 weeks, respectively) and 19 sham-operated rats $(5,6$ and 8 vehicle-treated rats for 2, 4 and 8 weeks, respectively).

Effects of YM358 on Hemodynamics in MI Rats Data are shown in Table 1 . HR significantly decreased 4 and 8 weeks after MI surgery, but recovered in YM358 treated MI rats 8 weeks after the coronary ligation. Loss of LV contractility was apparent from 2 weeks after MI surgery, as indicated by the decreases in LVSP and LV $d P / d t_{\max }$ in vehicletreated MI rats compared to sham-operated rats. Moreover, LVEDP significantly increased in these rats, indicating that LV failure had occurred 2 to 8 weeks after MI operation. Oral administration of YM358 $(30 \mathrm{mg} / \mathrm{kg} / \mathrm{d})$ for 8 weeks significantly attenuated the elevated LVEDP and LVSP in MI rats, although $\mathrm{LV} d P / d t_{\max }$ was not different from that in the vehicle-treated MI rats. In contrast, the decreased LVSP and $\mathrm{LV} d P / d t_{\max }$ and the increased LVEDP deteriorated following the treatment with YM358 2 weeks after the ligation. The decreases of SBP, DBP and MBP were significant 2, 4 and 8 weeks after the ligation in vehicle- or YM358-treated MI rats compared to sham-operated rats, but no significant difference was confirmed. YM358 further lowered the blood pressure in MI rats 2 to 8 weeks after MI surgery.

Effects of YM358 on Cardiac Hypertrophy in MI Rats Data are shown in Table 2. MI surgery significantly decreased body weight (BW). YM358 tended to increase the decreased $\mathrm{BW}$ in $\mathrm{MI}$ rats, and $\mathrm{BW}$ in MI rats did not significantly differ from that in sham-operated rats after 8-week treatment of YM358. LV weight in MI rats significantly decreased 2 weeks after the surgery, although it was not different from that of sham-operated rats either 4 or 8 weeks after the surgery. LV weight per BW (LV/BW) ratio in MI rats was not different from that in sham-operated rats 2 weeks after the surgery, but it significantly increased 4 and 8 weeks after

Table 1. Effects of YM358 (30 mg/kg/d) after 2-, 4-, and 8-Week Administration on Hemodynamic Parameters in MI Rats

\begin{tabular}{|c|c|c|c|c|c|c|c|c|c|}
\hline \multirow[b]{2}{*}{$\begin{array}{c}\text { Treatment } \\
n\end{array}$} & \multicolumn{3}{|c|}{2 weeks } & \multicolumn{3}{|c|}{4 weeks } & \multicolumn{3}{|c|}{8 weeks } \\
\hline & $\begin{array}{c}\text { Sham } \\
\text { Vehicle } \\
5\end{array}$ & $\begin{array}{c}\text { MI } \\
\text { Vehicle } \\
5\end{array}$ & $\begin{array}{c}\text { MI } \\
\text { YM358 } \\
6\end{array}$ & $\begin{array}{c}\text { Sham } \\
\text { Vehicle } \\
6\end{array}$ & $\begin{array}{c}\text { MI } \\
\text { Vehicle } \\
9\end{array}$ & $\begin{array}{c}\text { MI } \\
\text { YM358 } \\
7\end{array}$ & $\begin{array}{c}\text { Sham } \\
\text { Vehicle } \\
8\end{array}$ & $\begin{array}{c}\text { MI } \\
\text { Vehicle } \\
11\end{array}$ & $\begin{array}{c}\text { MI } \\
\text { YM358 } \\
13\end{array}$ \\
\hline HR (beats/min) & $386 \pm 3$ & $382 \pm 14$ & $368 \pm 9$ & $399 \pm 9$ & $364 \pm 8^{\#}$ & $386 \pm 11$ & $390 \pm 5$ & $347 \pm 8^{\#}$ & $389 \pm 7^{*}$ \\
\hline $\mathrm{SBP}(\mathrm{mmHg})$ & $130 \pm 3$ & $108 \pm 5^{\#}$ & $97 \pm 3^{\#}$ & $148 \pm 4$ & $119 \pm 4^{\#}$ & $106 \pm 5^{\#, *}$ & $138 \pm 2$ & $118 \pm 5^{\#}$ & $110 \pm 3^{\#}$ \\
\hline DBP (mmHg) & $101 \pm 2$ & $91 \pm 4^{\#}$ & $80 \pm 3^{\#, *}$ & $115 \pm 4$ & $97 \pm 3^{\#}$ & $82 \pm 3^{\#, *}$ & $110 \pm 2$ & $94 \pm 5^{\#}$ & $84 \pm 2^{\#, *}$ \\
\hline MBP (mmHg) & $111 \pm 3$ & $96 \pm 4^{\#}$ & $85 \pm 3^{\#, *}$ & $126 \pm 4$ & $104 \pm 4^{\#}$ & $90 \pm 3^{\#, *}$ & $119 \pm 2$ & $102 \pm 5^{\#}$ & $93 \pm 2^{\#, *}$ \\
\hline LVSP (mmHg) & $129 \pm 6$ & $110 \pm 5^{\#}$ & $96 \pm 2^{\#}$ & $164 \pm 7$ & $131 \pm 4^{\#}$ & $122 \pm 3^{\#}$ & $150 \pm 3$ & $132 \pm 4^{\#}$ & $119 \pm 4^{\#, *}$ \\
\hline LVEDP (mmHg) & $6 \pm 2$ & $19 \pm 3^{\#}$ & $23 \pm 2^{\#}$ & $2 \pm 1$ & $18 \pm 2^{\#}$ & $17 \pm 4^{\#}$ & $3 \pm 0$ & $21 \pm 4^{\#}$ & $10 \pm 2 *$ \\
\hline $\begin{array}{l}\mathrm{LV} d P / d t_{\max } \\
\quad(\times 10 \mathrm{mmHg} / \mathrm{s})\end{array}$ & $1263 \pm 85$ & $845 \pm 45^{\#}$ & $730 \pm 20^{\#}$ & $1475 \pm 86$ & $966 \pm 68^{\#}$ & $914 \pm 84^{\#}$ & $1175 \pm 48$ & $842 \pm 70^{\#}$ & $829 \pm 52^{\#}$ \\
\hline
\end{tabular}

Values are means \pm S.E.M. HR, heart rate; SBP, systolic blood pressure; DBP, diastolic blood pressure; MBP, mean blood pressure; LVSP, left ventricular systolic pressure; LVEDP, left ventricular end-diastolic pressure; LV $d P / d t_{\max }$, first derivative of left ventricular pressure. \# $p<0.05 v s$. sham-operated rats, $* p<0.05 v s$. vehicle-treated MI rats. 
Table 2. Effects of YM358 (30 mg/kg/d) after 2-, 4-, and 8-Week Administration on Body Weight, Cardiac Weight, and Lung Weight in MI Rats

\begin{tabular}{|c|c|c|c|c|c|c|c|c|c|}
\hline \multirow[b]{2}{*}{$\begin{array}{c}\text { Treatment } \\
n\end{array}$} & \multicolumn{3}{|c|}{2 weeks } & \multicolumn{3}{|c|}{4 weeks } & \multicolumn{3}{|c|}{8 weeks } \\
\hline & $\begin{array}{l}\text { Sham } \\
\text { Vehicle } \\
5\end{array}$ & $\begin{array}{c}\text { MI } \\
\text { Vehicle } \\
5\end{array}$ & $\begin{array}{c}\text { MI } \\
\text { YM358 } \\
6\end{array}$ & $\begin{array}{c}\text { Sham } \\
\text { Vehicle } \\
6\end{array}$ & $\begin{array}{c}\text { MI } \\
\text { Vehicle } \\
9\end{array}$ & $\begin{array}{c}\text { MI } \\
\text { YM358 } \\
7\end{array}$ & $\begin{array}{l}\text { Sham } \\
\text { Vehicle } \\
8\end{array}$ & $\begin{array}{c}\text { MI } \\
\text { Vehicle } \\
11\end{array}$ & $\begin{array}{c}\text { MI } \\
\text { YM358 } \\
13\end{array}$ \\
\hline BW (g) & $307 \pm 9$ & $271 \pm 8^{\#}$ & $253 \pm 10^{\#}$ & $351 \pm 11$ & $314 \pm 10^{\#}$ & $304 \pm 11^{\#}$ & $365 \pm 9$ & $335 \pm 10^{\#}$ & $347 \pm 5$ \\
\hline $\mathrm{LV}(\mathrm{mg})$ & $606 \pm 24$ & $523 \pm 19^{\#}$ & $464 \pm 22^{\#}$ & $635 \pm 11$ & $624 \pm 25$ & $554 \pm 21^{\#, *}$ & $654 \pm 8$ & $646 \pm 22$ & $608 \pm 9^{\#}$ \\
\hline RV (mg) & $163 \pm 12$ & $237 \pm 13^{\#}$ & $239 \pm 9^{\#}$ & $161 \pm 4$ & $304 \pm 16^{\#}$ & $255 \pm 27^{\#}$ & $169 \pm 4$ & $351 \pm 27^{\#}$ & $248 \pm 23^{\#, *}$ \\
\hline LA (mg) & $22 \pm 3$ & $64 \pm 17^{\#}$ & $58 \pm 5^{\#}$ & $18 \pm 2$ & $60 \pm 5^{\#}$ & $49 \pm 8^{\#}$ & $18 \pm 2$ & $75 \pm 15^{\#}$ & $42 \pm 7 *$ \\
\hline RA (mg) & $47 \pm 7$ & $106 \pm 14^{\#}$ & $102 \pm 4^{\#}$ & $58 \pm 5$ & $105 \pm 9^{\#}$ & $92 \pm 17$ & $65 \pm 6$ & $146 \pm 18^{\#}$ & $84 \pm 9 *$ \\
\hline Lung weight (mg) & $1241 \pm 246$ & $2393 \pm 297^{\#}$ & $2986 \pm 169^{\#}$ & $1096 \pm 25$ & $2491 \pm 191^{\#}$ & $2028 \pm 290^{\#}$ & $1102 \pm 12$ & $2497 \pm 268^{\#}$ & $1815 \pm 236^{\#, *}$ \\
\hline $\mathrm{LV} / \mathrm{BW}(\mathrm{mg} / \mathrm{g})$ & $1.98 \pm 0.09$ & $1.93 \pm 0.03$ & $1.84 \pm 0.06$ & $1.81 \pm 0.03$ & $1.99 \pm 0.03^{\#}$ & $1.82 \pm 0.02 *$ & $1.80 \pm 0.02$ & $1.93 \pm 0.03^{\#}$ & $1.75 \pm 0.03 *$ \\
\hline $\mathrm{RV} / \mathrm{BW}(\mathrm{mg} / \mathrm{g})$ & $0.53 \pm 0.04$ & $0.88 \pm 0.06^{\#}$ & $0.96 \pm 0.06^{\#}$ & $0.46 \pm 0.01$ & $0.98 \pm 0.07^{\#}$ & $0.86 \pm 0.12^{\#}$ & $0.47 \pm 0.01$ & $1.07 \pm 0.09^{\#}$ & $0.73 \pm 0.08^{\#, *}$ \\
\hline $\mathrm{LA} / \mathrm{BW}(\mathrm{mg} / \mathrm{g})$ & $0.07 \pm 0.01$ & $0.24 \pm 0.07^{\#}$ & $0.23 \pm 0.02^{\#}$ & $0.05 \pm 0.00$ & $0.19 \pm 0.02^{\#}$ & $0.17 \pm 0.03^{\#}$ & $0.05 \pm 0.01$ & $0.23 \pm 0.05^{\#}$ & $0.12 \pm 0.02 *$ \\
\hline $\mathrm{RA} / \mathrm{BW}(\mathrm{mg} / \mathrm{g})$ & $0.16 \pm 0.02$ & $0.39 \pm 0.05^{\#}$ & $0.41 \pm 0.03^{\#}$ & $0.17 \pm 0.01$ & $0.34 \pm 0.04^{\#}$ & $0.32 \pm 0.06^{\#}$ & $0.18 \pm 0.02$ & $0.45 \pm 0.06^{\#}$ & $0.25 \pm 0.03 *$ \\
\hline $\begin{array}{l}\text { Lung weight/BW } \\
(\mathrm{mg} / \mathrm{g})\end{array}$ & $4.08 \pm 0.87$ & $8.93 \pm 1.21^{\#}$ & $12.01 \pm 1.12^{\#}$ & $3.14 \pm 0.09$ & $8.07 \pm 0.77^{\#}$ & $6.92 \pm 1.19^{\#}$ & $3.02 \pm 0.03$ & $7.56 \pm 0.83^{\#}$ & $5.35 \pm 0.76^{\#, *}$ \\
\hline
\end{tabular}

Values are means \pm S.E.M. BW, body weight; LV, left ventricle; RV, right ventricle; LA, left atrium; RA, right atrium. $\# p<0.05 v s$. sham-operated rats, $* p<0.05 v s$. vehicletreated MI rats.

the surgery. YM358 significantly attenuated the increase of $\mathrm{LV} / \mathrm{BW}$ ratio in MI rats, and LV/BW ratio reached the level close to that in sham-operated rats 4 and 8 weeks after the surgery. RV weight, LA weight, RA weight, lung weight, and their ratios to $\mathrm{BW}$ (RV/BW, LA/BW, RA/BW, and Lung/BW) in MI rats significantly increased compared with those in sham-operated rats after 2 to 8 weeks treatment of YM358. $\mathrm{RV}$ weight and RV/BW ratio in MI rats increased time-dependently. LA weight, RA weight, LA/BW ratio and RA/BW ratio in MI rats increased time-dependently from 4 weeks after the surgery. Oral administration of YM358 to MI rats tended to decrease RV/BW, LA/BW, RA/BW and Lung/BW ratios time-dependently. Eight weeks after the surgery, YM358 significantly decreased these increased ratios in MI rats.

\section{DISCUSSION}

In heart failure, a number of neurohormonal mechanisms, such as RAS and the sympathetic nervous system, are activated to compensate for impaired cardiac function. Because ischemic heart disease is the most common contemporary cause of heart failure, we investigated the effects of chronic treatment with YM358 on the development of cardiac hypertrophy and dysfunction due to MI in rats. MI rats consistently showed signs of cardiac dysfunction compared to sham-operated rats: decreases in LVSP and LV $d P / d t_{\max }$, which are parameters of cardiac contractility and caused by CHF, and increase in LVEDP, which is induced by CHF. Based on the measurement of time course changes of hemodynamic parameters in rats, we confirmed that cardiac dysfunction could already be ascertained 2 weeks after MI surgery.

It is reported that plasma renin activity increases in the earlier phase ${ }^{20)}$ and the chronic phase ${ }^{21,22)}$ of MI. Moreover, MI increases ACE activity and Ang II levels in plasma of rats 3,7 , and 28 days after infarction. ${ }^{23)}$ From these findings, it was believed that RAS was activated immediately to compensate for the reduction of cardiac contractility from acute phase to chronic phase, and that $\mathrm{AT}_{1}$ receptor antagonist might have blunted the activated RAS from the early reactive phase to chronic phase after MI. In this study, YM358 low- ered the decreased SBP, DBP and MBP in MI rats. YM358 showed a hypotensive effect in rats with CHF from the early phase to chronic phase after MI surgery. Moreover, LVSP and LVEDP were lowered in MI rats after 8-week treatment with YM358. Therefore, long-term treatment with YM358 was believed to improve hemodynamics in rats with CHF.

Regarding the cardiac index, RV hypertrophy was indicated from 2 weeks after MI surgery, and LV hypertrophy from 4 weeks. LV weight in MI rats was significantly lower than that in sham-operated rats 2 weeks after the surgery. The infarcted area of LV was replaced by a thin fibrous tissue. From 4 weeks after the surgery, the un-infarcted area was hypertrophied. In the present study, daily administration of YM358 prevented cardiac hypertrophy time-dependently. Our result was supported by some reports describing that $\mathrm{AT}_{1}$ receptor antagonist prevented cardiac hypertrophy. ${ }^{24,25)}$ On the other hand, there is another report that $\mathrm{AT}_{1}$ receptor antagonist does not prevent cardiac hypertrophy. ${ }^{26)}$ The discrepancies between these results in preventing cardiac hypertrophy might be due to the difference in treatment periods. Administrations of $\mathrm{AT}_{1}$ receptor antagonist, L-158809 for 2 months $^{24)}$ and losartan for 6 weeks ${ }^{25)}$ prevent cardiac hypertrophy in MI rats, while that of losartan for 2 weeks does not. ${ }^{26)}$ Indeed, 2-week administration of YM358, in the present study, did not prevent cardiac hypertrophy in MI rats. However, 8-week administration of YM358 significantly decreased cardiac hypertrophy in MI-induced CHF rats. Other researchers also report that losartan attenuates LV hypertrophy after 4-week treatment accompanying the decrease of cardiac fibrosis, although it did not prevent LV hypertrophy or decrease cardiac fibrosis after 2-week treatment. ${ }^{27)}$ Cardiac remodeling after MI is related to the increase of cardiac fibrosis, and Ang II is thought to be one of the stimulants of cardiac fibrosis in post-MI. In the present study, although we did not ascertain the morphological changes in post-MI heart, one probable reason why the long-term treatment with YM358 attenuated cardiac hypertrophy was that it might blunt the progression of cardiac fibrosis in CHF rats.

We previously reported that YM358 decreased cardiac hypertrophy in aortocaval shunt rats without affecting blood pressure. ${ }^{18)}$ The finding indicated that YM358 would directly 
influence cardiac hypertrophy in rats with chronic heart failure. Although we used the same dose $(30 \mathrm{mg} / \mathrm{kg})$ of YM358 as used in the previous study, YM358 lowered MBP and DBP during this experiment, and consequently decreased the afterload in MI-induced CHF rats when attenuation of cardiac hypertrophy due to the treatment of YM358 was observed. These findings suggested that the inhibitory effect of YM358 on progression of cardiac hypertrophy might be caused by an indirect action through the improvement of hemodynamics as well as a direct action on myocyte. However, there has been a report that differences in the antihypertensive therapy do not always attenuate LV hypertrophy. ${ }^{28)}$ The expression of cardiac $\mathrm{AT}_{1}$ receptor mRNA in MI rats is 2.2 times that in sham-operated rats, 7 weeks after the surgery. ${ }^{14)}$ In MI rats, 6-week treatment with losartan and an ACE inhibitor of captopril attenuates the increase of cardiac $\mathrm{AT}_{1}$ receptor mRNA expression. ${ }^{29)}$ These reports suggest that the inhibitory effect of YM358 on progression of cardiac hypertrophy in MI rats would be caused by a direct action to $\mathrm{AT}_{1}$ receptor on myocyte. Moreover, 8-week treatment with YM358 significantly decreased the elevated LVEDP in MI rats and consequently these values did not statistically differ from those in sham-operated rats. The decrease of LVEDP in MI rats was accompanied by the significant decrease of $\mathrm{LV} / \mathrm{BW}$ and LA/BW ratios. These results suggested that prevention of cardiac hypertrophy might improve or maintain cardiac functions after MI.

It is well known that activation of RAS in CHF brings about an enhancement of vascular tonus by increasing peripheral resistance and body fluid retention due to the secretion of aldosterone and vasopressin, or direct action on kidneys. When RAS activation persists for a long period in $\mathrm{CHF}$, it leads to increases in preload and afterload. Thereafter, it results in lung congestion and a fall in cardiac output, and consequently the condition of heart failure deteriorates. Eight-week administration of YM358 to MI-induced CHF rats tended to decrease the weight of lung and the lung weight/BW ratio which defined the degree of pulmonary congestion in the present study. Such reductions of lung weight and lung weight/BW ratio after administration of YM358 were in agreement with the depression of cardiac hypertrophy and the improvement of hemodynamics. In our preliminary experiments, the diuretic effect of YM358 was shown in saline-loaded SHR. Consequently, the decreases of pulmonary congestion and LVEDP after long-term YM358 treatment might be explained in part by its diuretic effect in MI rats.

In summary, the results of the present study indicated that chronic treatment with YM358 prevented MI-induced cardiac hypertrophy, lung congestion, and LVEDP elevation, and consequently maintained the cardiac function. YM358 might be useful in the treatment of CHF patients after ischemic heart disease.

\section{REFERENCES}

1) Schunkert H., Tang S. S., Litwin S. E., Diamant D., Riegger G., Dzau V. J., Ingelfinger J. R., Cardiovasc. Res., 27, 731-735 (1993).

2) Luchner A., Stevens T. L., Borgeson D. D., Redfield M. M., Bailey J. E., Sandberg S. M., Heublein D. M., Burnett J. C., Jr., Hypertension, 28, 472-477 (1996).

3) Neyses L., Nouskas J., Luyken J., Fronhoffs S., Oberdorf S., Pfeifer U., Williams R. S., Sukhatme V. P., Vetter H., J. Hypertens., 11, 927-934 (1993).

4) Schunkert H., Sadoshima J., Cornelius T., Kagaya Y., Weinberg E. O., Izumo S., Riegger G., Lorell B. H., Circ. Res., 76, 489— 497 (1995).

5) Weber K. T., Janicki J. S., Br. J. Clin. Pharmacol., 28, 141S-150S (1989).

6) Smits J. F., Passier R. C., Nelissen-Vrancken H. J., Cleutjens J. P., Kuizinga M. C., Daemen M. J., Eur. Heart J., 16 (Suppl.), N46-51 (1995).

7) Verme-Gibboney C., Am. J. Health-Sys. Pharm., 54, 2689-2703 (1997).

8) Richer C., Fornes P., Cazaubon C., Domergue V., Nisato D., Giudicelli J. F., Cardiovasc. Res., 41, 100-108 (1999).

9) Zhu B., Sun Y., Sievers R. E., Browne A. E., Pulukurthy S., Sudhir K., Lee R. J., Chou T. M., Chatterjee K., Parmley W. W., J. Am. Coll. Cardiol., 35, 787-795 (2000).

10) Pfeffer M. A., Annu. Rev. Med., 46, 455-466 (1995).

11) Pfeffer M. A., Pfeffer J. M., Fishbein M. C., Fletcher P. J., Spadaro J., Kloner R. A., Braunwald E., Circ. Res., 44, 503-512 (1979).

12) Teerlink J. R., Goldhaber S. Z., Pfeffer M. A., Am. Heart J., 121, 1852-1853 (1991)

13) Stassen F. R., Willemsen M. J., Janssen G. M., Fazzi G. E., Schiffers P. M., Smits J. F., De Mey J. G., Br. J. Pharmacol., 120, 1505-1512 (1997).

14) Zhang G., Yang Y., Pu S., Shen X., Peng T., Chen H., Chinese Medical J., 112, 593-596 (1999).

15) Shibasaki M., Fujimori A., Takanashi M., Kusayama T., Tokioka T., Satoh Y., Okazaki T., Uchida W., Inagaki O., Yanagisawa I., Eur. J. Pharmacol., 335, 167-173 (1997).

16) Shibasaki M., Fujimori A., Kusayama T., Tokioka T., Satoh Y., Okazaki T., Uchida W., Inagaki O., Yanagisawa I., Eur. J. Pharmacol., 335, 175-184 (1997).

17) Yamaguchi N., Fujimoto K., Fujii T., Suzuki T., Kawashima K., Jpn. J. Pharmacol., 73, 83-91 (1997).

18) Tokioka-Akagi T., Fujimori A., Shibasaki M., Inagaki O., Uchida W., Yanagisawa I., Jpn. J. Pharmacol., 86, 79-85 (2001).

19) Yamagishi H., Kim S., Nishikimi T., Takeuchi K., Takeda T., J. Mol. Cell. Cardiol., 25, 1369-1380 (1993).

20) Hirsch A. T., Opsahl J. A., Lunzer M. M., Katz S. A., Am. J. Physiol., 276, H1818-H1826 (1999).

21) Kohzuki M., Kanazawa M., Yoshida K., Tsutsumi E., Yasujima M., Abe K., Johnston C. I., Sato T., Clin. Sci., 90, 31-36 (1996).

22) Burrell L. M., Phillips P. A., Risvanis J., Chan R. K., Aldred K. L., Johnston C. I., Am. J. Physiol., 275, H176-H182 (1998).

23) Duncan A. M., Burrell L. M., Kladis A., Campbell D. J., J. Card. Fail., 3, 41-52 (1997).

24) Liu Y. H., Yang X. P., Sharov V. G., Nass O., Sabbah H. N., Peterson E., Carretero O. A., J. Clin. Invest., 99, 1926-1935 (1997).

25) Schieffer B., Wirger A., Meybrunn M., Seitz S., Holtz J., Riede U. N., Drexler H., Circulation, 89, 2273-2282 (1994).

26) Raya T. E., Fonken S. J., Lee R. W., Daugherty S., Goldman S., Wong P. C., Timmermans P. B., Morkin E., Am. J. Hypertens., 4, 334S$340 \mathrm{~S}(1991)$

27) Ju H., Zhao S., Jassal D. S., Dixon I. M., Cardiovasc. Res., 35, 223 232 (1997).

28) Tsotetsi O. J., Woodiwiss A. J., Netjhardt M., Qubu D., Brooksbank R., Norton G. R., Hypertension, 38, 846-851 (2001).

29) Zhang G. X., Pu S. Y., Yang Y. Z., Shen X. D., Peng T. Q., Chen H. Z., Chung-Kuo Yao Li Hsueh Pao-Acta Pharmacologica Sinica, 18, 431434 (1997). 